\title{
The Relationship among Negative Peer Pressure, Drinking alcohol and Early sexual engagement among secondary school students' in Assosa Zone, West of Ethiopia
}

Demelash Wubet ( $\square$ amandemelash2009@gmail.com )

Woldia University

\section{Research Article}

Keywords: Negative peer pressure, Drinking alcohol, early sexual engagement

Posted Date: March 11th, 2021

DOI: https://doi.org/10.21203/rs.3.rs-275610/v1

License: (9) This work is licensed under a Creative Commons Attribution 4.0 International License.

Read Full License 


\section{Abstract}

Background: Sexual and reproductive health problems are the main causes of death, psychological abnormality, disability, and disease among young people in the world particularly in Ethiopia. Adolescents represent one of the groups at highest risk for HIV infection.

Objectives: Examined the relationship among negative peer pressure, drinking alcohol and early sexual engagement.

Methods: This study employed correlation research design and closed - ended questionnaires for 355 randomly selected (177 females and 178 males).

Results: Negative peer pressure was significantly correlated with drinking alcohol and early sexual engagement $(r=-.193, p=.010)$ and $(r=.167, p=.006)$ respectively. Additionally, drinking alcohol also was correlated significantly and positively with early sexual engagement $(r=.151, p=.000)$. But, there was no statistically significant gender difference on negative peer pressure, drinking alcohol and early sexual engagement $(p>0.05)$. The variables, negative peer pressure and drinking alcohol jointly contributed .532 $(53.2 \%)$ of the variance on early sexual engagement $(p<0.05)$. Relative contribution of negative peer pressure and drinking alcohol on early sexual engagement is-.362 (36.2\%) and-.454 (45.4\%) respectively.

Conclusions: as negative peer pressure increases students' drinking alcohol and early sexual engagement also increases. Similarly, when drinking alcohol increases students' early sexual engagement also increases. Both negative peer pressure and drinking alcohol were the contributing factors of early sexual engagement.

\section{Introduction}

This study was geographically delimited in Assosa Zone, West of Ethiopia. The study addressed the relationship among negative peer pressure, drinking alcohol and early sexual engagement among secondary school students. Peer pressure is social influence exerted a power on individuals to change his/ her attitudes, values or behaviors [1]. Peer pressure is a more dominant factor for many adolescents, because it creates and maintains a culture separate from home in which they raised [2]. Furthermore, negative peer pressure plays an increasingly important role in encouraging age mates by showing undesirable activities [3]. According to [5] 25(17.2\%) respondents involved in premarital sexual practice and unsafe sexual intercourse by the negative pressure of their peers. Moreover, in negative peer pressure when close friends smoke cigarettes, drink alcohol, use illegal drugs and sexually active, sooner or later the adolescent is likely to do these things too [5]. In addition, peer group pressure was the main motivating forces that made students to engage in sexual activities followed by self-initiation and pornographic films [6]. Furthermore, adolescents who use tobacco, alcohol, marijuana and illegal drugs are more likely to be sexually active to engage in risky sexual behavior and to experience its consequences such as accidental pregnancy or contracting a sexually transmitted disease-than those who do not use these substances [4]. Negative peer pressure is the most important factor that 
precipitated the first sexual encounter [8]. Risky sexual behavior is significantly associated with perception of peers' involvement in sexual intercourse [9].

In addition, the study conducted in northern Ethiopia showed that $83.3 \%$ respondents smoke cigarette, $77.8 \%$ drink alcohol and $81.1 \%$ chew chat because of their peer group influences [10].

Even though, research has been conducted by [5] on negative peer pressure; [11] on relationship among peer group influences; alcohol consumption and academic self esteem and [12] on the relationship between peer group pressure and responsibility behavior. Moreover, [3] also conducted research on family and peer influences on adolescent behavior. Besides, the above mentioned studies didn't assess the relationship among negative peer pressure, drinking alcohol and early sexual engagement among secondary school student. Moreover, there was no comprehensive and current research on the relationship among negative peer pressure, drinking alcohol and early sexual engagement on secondary school students at Assosa Zone, West of Ethiopia. Hence, this study intends to fill the gap especially with focus to examine the relationship among negative peer pressure, drinking alcohol and early sexual engagement of secondary school students.

\section{Aims of the Study}

The main aim of this study was to examine the relationship among negative peer pressure, drinking alcohol and early sexual engagement among secondary school students. Specifically to:

1. Examine the relationship between negative peer pressure and drinking alcohol.

2. Investigate the relationship between negative peer pressure and early sexual engagement.

3. Find out the relationship between drinking alcohol and early sexual engagement.

4. Examine gender difference in negative peer pressure, drinking alcohol and early sexual engagement.

5. Predict the significant relative contribution of negative peer pressure and drinking alcohol on student early sexual engagement?

\section{Methods}

\section{Design of the Study}

This study is mainly a quantitative study and it follows a correlation research design. A correlation research design is a study that describes the extent to which the variables are related. Correlation method tries to determine whether and to what degree a relationship exists between two or more variables. The present study examined the relationship among negative peer pressure and drinking alcohol; negative peer pressure and early sexual engagement; drinking alcohol and early sexual engagement.

\section{Study Area}


The study area was Asosa Zone, West of Ethiopia. The study covered three selected Woredas namely, Assosa Woreda, Bambasi Woreda and Homosha Woreda. In Assosa Woreda the included schools were Nebarkomeshga and Hoha number two; from Bambasi Woreda;Bambasi and Keshemando and from Homosha Woreda;Homosha and Tsorealmatema secondary schools were selected.

\section{Population}

The target population of the study was all students enrolled in grade 9 and 10 in secondary schools of Assosa Zone, West of Ethiopia in 2012 E.C academic year. There were totally 3,165 students in the selected schools of which 1,901 were males and 1,264 were females.

\section{Sample Size}

The sample size was determined by using Yamane's formula [17].

$\mathrm{n}=\mathrm{N} / 1+\mathrm{N}(\mathrm{e})^{2}$

Where, $\mathrm{N}=$ Total population

$\mathrm{n}=$ Sample size

$\mathrm{e}=$ Sampling error $(5 \%)$

$\mathrm{n}=\mathrm{N} / 1+\mathrm{N}(\mathrm{e})^{2}$

$\mathrm{n}=3165 / 1+3165(0.05)^{2}$

$n=355$

$\mathrm{n} 1($ Female $)=50 \%=355 \times 50 / 100=178$ and $\mathrm{n} 2$ (Male) $=50 \%=355 \times 50 / 100=177$

\section{Sampling Techniques}

The researcher used multi stage sampling method. Purposive sampling was used to select three Woredas among the 7 Woredas from Assosa Zone due to accessibility of transportation and nearness. Using a list of all the secondary schools (15) in the selected Zone 6 (40\%) secondary schools were randomly selected. The selected schools were stratified by their grade level (grade 9 and 10). Then, the grade levels were stratified by section. And the sections were stratified by sex. Using Proportionate stratified random sampling 180 male and 175 female students were selected from sample size 355 . It is to ensure equal representation of boys and girls in the study.

\section{Data Collection Tools}

The instruments were structured questionnaires in the form of self inventory reports in order to gain a comprehensive input which reflects the right feeling of respondents. To measure the negative peer 
pressure, the Peer Pressure Inventory was used. This instrument was developed from [1]. This instrument is a 21-paired item. But, for the purposes of this study the negative peer pressure items were taken. The scores of respondents for each item were coded from $-3-1$ with the no influence option scored as zero. Thus: $0=$ no negative peer pressure, $-1=$ little negative peer pressure, $-2=$ medium negative peer pressure and $-3=$ high negative peer pressure.

Drinking Alcohol to measure drinking alcohol, Rutgers Alcohol Problem Index was employed. Rutgers Alcohol Problem Index is a 10-item (modified) self-administered instrument for assessing adolescent alcohol consumption. It was developed by [8] Rutgers Alcohol Problem Index measures adolescents' problems as a result of drinking alcohol, the feeling and motivation to use alcohol and any further information about the intensity and its influence on individuals early sexual engagement of each item were coded from 0 to 3 where, 0 represented none, 1 represented 1-2 times, 2 represented 3-5 times and 3 represented more than 5 times. Additionally the sexual engagement questionnaires were constructed by the researcher himself and edited by research and community service board members. The questionnaire has 6 items which was coded in $1=$ yes and $2=$ no alternative form.

\section{Reliability and Validity of the instrument}

The researcher used a pilot testing. The purpose of this pilot testing was to get comment and information about the clarity and understandability of the questionnaires. In addition, this pilot testing helped to avoid vague words and restructure the actual questionnaires. The researcher adapted adequate questions considering the context of the target setting. The standardized English version scales were translated in to Amharic version which is a local language of the participants in order to be more understandable, and to gain valid and more reliable data.

\section{Data Analysis Methods}

The researcher used descriptive statistics to describe the demographic characteristics of the participants. Pearson product moment correlation coefficient was used to examine the relationship between (negative peer pressure and drinking alcohol, negative peer pressure and early sexual engagement, drinking alcohol and early sexual engagement). In addition, independent sample t-test was employed to test whether there is significant difference in negative peer pressure, drinking alcohol and early sexual engagement across gender. Moreover, multiple regression analysis was used to test the relative contribution of negative peer pressure and drinking alcohol on early sexual engagement. Additionally, the analysis was done by using SPSS version 20 .

\section{Data Analysis Procedures}

First the quantitative data gathered by questionnaires was collected, counted and organized according to its structure and type. Then it was inserted in to computer software especially using statistical package for social sciences (SPSS) version 20. Then it was analyzed, interpreted and discussed referencing the research objectives. 


\section{Results}

\section{Characteristics of the participants}

In this study the total number participants were 355 of which $177(49.9 \%)$ were males and $178(50.1 \%)$ were female respondents and 149 (40.9\%) and 206 (56.6\%) respondents were grade nine and grade ten respectively. The minimum and maximum age of the participants was 14 and 23 years respectively. But, the majority of the participants 329 (90.4\%) were between 14 and 17 means that under 18 years.

\section{The Relationship among variables}

Table 1 Pearson Correlation among Negative peer pressure, Drinking alcohol and Early sexual engagement $(N=355)$

\begin{tabular}{|llll|}
\hline Variables & Negative Peer pressure & Drinking Alcohol & Early Sexual Engagement \\
\hline Negative Peer pressure & 1 & $.193^{\star}$ & $.167^{\star \star}$ \\
Drinking Alcohol & $.193^{\star}$ & $(.011)$ & $(.006)$ \\
& $(.010)$ & 1 & $.151^{\star \star}$ \\
Early Sexual Engagement & $.167^{\star *}$ & & $(.000)$ \\
& $(.006)$ & $.151^{\star \star}$ & 1 \\
\hline
\end{tabular}

*. Correlation is significant at the 0.05 level (2-tailed).

**. Correlation is significant at the 0.01 level (2-tailed).

As observed, on table 1 the result of this study indicated that negative peer pressure is significantly and positively correlated with students' drinking alcohol $(r=-.193, p=.010)$. This means that as negative peer pressure increases students' drinking alcohol also increases in the target setting. Table 1 also shows that, there is significant and positive relationship between students' negative peer pressure and early sexual engagement $(r=.167, p=.006)$. This result indicated that as negative peer pressure increases students' early sexual engagement also increases. Again as shown on table 1, there is a statistically significant positive relationship between students' drinking alcohol and early sexual engagement $(r=.151$, $\mathrm{p}=.000$ ). This means that as drinking alcohol increases students' early sexual engagement also increases. 
Table 2 Independent sample t-test group statistics ( $N=355)$

\begin{tabular}{|llllllll|}
\hline Variables & Sex & $\mathrm{N}$ & Mean & $\begin{array}{l}\text { Std. } \\
\text { deviation }\end{array}$ & DF & T & $\begin{array}{l}\text { Sig. (2 } \\
\text { tailed) }\end{array}$ \\
\hline Negative Peer pressure & Male & 177 & 52.05 & 8.041 & 353 & -.091 & .077 \\
\cline { 2 - 8 } & Female & 178 & 51.97 & 8.243 & 353 & -.091 & .077 \\
\hline Drinking Alcohol & Male & 177 & 30.89 & 5.453 & 353 & .772 & .927 \\
\cline { 2 - 9 } & Female & 178 & 29.86 & 5.486 & 353 & .772 & .927 \\
\hline $\begin{array}{l}\text { Early sexual } \\
\text { Engagement }\end{array}$ & Male & 177 & 33.98 & 9.363 & 353 & .653 & .723 \\
\hline
\end{tabular}

*. No significant gender difference at the 0.05 level (2-tailed).

**. No significant gender difference at the 0.01 level (2-tailed).

As depicted, on the above table 2, the result indicated that there was no significant gender difference in negative peer pressure of male $(M=52.05, S D=8.04, D F=353, T=-0.91)$ and female $(M=51.97, S D=$ 8.24, $\mathrm{DF}=353$ and $\mathrm{T}=-0.91, \mathrm{p}>0.05)$. Moreover, there was no also statistically significant gender difference in drinking alcohol of male $(M=30.89, S D=5.453, D F=353, T=.772$ and $P>0.05)$ and female $(M=29.86, S D=5.486, D F=353, T=.772$ and $P>0.05)$. Additionally, the above table 2 depicted that there was no statistically significant difference in early sexual engagement of male $(M=33.98, S D=$ 9.363, $\mathrm{DF}=353, \mathrm{~T}=.653$ and $\mathrm{p}>0.05)$ and female $(\mathrm{M}=33.78, \mathrm{SD}=9.772), \mathrm{DF}=353, \mathrm{~T}=.723$ and $\mathrm{p}>$ 0.05 . On the above table 2 the independent sample t-test indicated that being male or female may not have significant differences on students' negative peer pressure, drinking alcohol and early sexual engagement.

\section{Multiple regression analysis}

Table 3 Relative Contribution of negative peer pressure and drinking alcohol on students' early sexual engagement $(\mathrm{N}=355)$ 


\begin{tabular}{|lcccccccc|}
\hline Variables & B & Beta & Std error & $R^{2}$ & $\operatorname{AdjR}^{2}$ & $T$ & Sig & $F$ \\
\hline Constant & & & & & & & & \\
\hline Negative peer pressure & 23.000 & & 4.848 & .44 & 0.532 & 12.45 & 0.00 & 18.33 \\
Drinking alcohol & -.143 & -.362 & .05965 & & & 4.091 & 0.021 \\
& & & & & & & & \\
& & & & & & & & \\
& -.632 & -.454 & .0976 & & & .354 & 0.038 \\
\hline
\end{tabular}

The multiple regression analysis result on table 3 above depicted that both negative peer pressure and drinking alcohol has statistically significant contribution to the prediction of students' early sexual engagement $(p<0.05)$. The variables, students' negative peer pressure and drinking alcohol jointly contributed .532 that is $53.2 \%$ of the variance in secondary school students' early sexual engagement. But the relative contribution of negative peer pressure and drinking alcohol for the variation of early sexual engagement is $-.362(36.2 \%)$ and $-.454(45.4 \%)$ respectively.

\section{Discussions}

This section discus results of the current study and examines if results are consistent with previous findings or not.

In a related study [13] found that, if adolescents' friends do not engaged in activities like alcohol consumption they are less likely to be involved in the group. According to the finding of [4], $94(23.4 \%)$ of adolescent students are under negative peer pressure. Moreover the discussant stated that using of substances (chewing chat, smoking cigarettes and 'shish', and drinking alcohol) and early sexual activities are common among secondary and preparatory school students. Furthermore, study conducted in northern Ethiopia showed that $83.3 \%$ respondents smoke cigarette, $77.8 \%$ drink alcohol and $81.1 \%$ chew chat because of their peer group influences [10]. Peer pressure have negative influences such as pressure to use illegal drugs, to show anti - social or undesirable behaviors [1]. In addition, peer pressures can encourage doing unacceptable activities: such as drinking alcohol, theft, engaging in sexual activities [4]. Negative peer pressure can aggravate adolescent's misbehaviors such as, participation in antisocial activities, using illegal drugs, involving in risky sexual activities [3]. There was strong association between peer pressure and early sexual activities, substance use and educational involvement that indicates peer pressure is the most common reason why secondary and preparatory school students use substance, indulge in early sexual activities [4]. There was statistically significant negative relationship between adolescents' peer group influence and alcohol consumption [11]. Similarly with the above findings the 
current study also found that negative peer pressure was significantly and positively correlated with students' drinking alcohol. Similarly, there was statistically significant and positive relationship between students' negative peer pressure and early sexual engagement. This indicated that as negative peer pressure increases students' early sexual engagement also increases in Assosa Zone selected secondary schools. In addition, there was statistically significant positive relationship between students' drinking alcohol and early sexual engagement. This means that as drinking alcohol increases students' early sexual engagement also increases. Concerning peer group influence [14], reported that there is consistent qualitative difference in boys' and girls' friendship in the early adolescent years, for instance girls' friendships are marked by greater intimacy, self disclosure and validation and caring than those of boys. Female adolescents are more exposed for peer groups' influence than male counter parts [15]. The present research finding is not consistent with the above report. Boys tend to engage in more action oriented pursuits and girls spend more time talking together [16]. According to [16] the type of activities in which males and females engaged differently in peer group influences, but in the present study lack of gender difference in negative peer pressure. Regarding drinking alcohol the finding of the present study revealed no gender difference. Similar with the findings of the present study [4] and [11] also found that there was no significant difference on the extent of peer pressure, drinking alcohol and involvement of early sexuality on both sexes.

\section{Conclusions}

This study concluded that negative peer pressure was significantly and positively correlated with students' drinking alcohol and early sexual engagement. Moreover, there was statistically significant positive relationship between students' drinking alcohol and early sexual engagement.

On the other hand, there was no statistically significant gender difference on students negative peer pressure, drinking alcohol and early sexual engagement $(p>0.05)$. The multiple regression analysis result depicted that both negative peer pressure and drinking alcohol has statistically significant contribution to the prediction of students' early sexual engagement $(p<0.05)$. The variables, students' negative peer pressure and drinking alcohol jointly contributed .532 that is $53.2 \%$ of the variance in early sexual engagement. The relative contribution of negative peer pressure and drinking alcohol for the variation of early sexual engagement is $-.362(36.2 \%)$ and $-.454(45.4 \%)$ respectively. The study recommended that, health workers, parents, school counselors, school directors and teachers should work together and discuss periodically about the side effect of negative peer pressure and drinking alcohol on early sexual engagement of students. Further research should be done on the relationship among negative peer pressure, drinking alcohol and early sexual engagement including the unaddressed regions of Ethiopia.

\section{Declarations}

\section{Ethics protocols}


The study protocol was approved by the Institutional Research Review committee of Assosa University (Reference number: ASU/RCS/144/2012). The institutional research review committee in Assosa University has guidelines and regulations how individual's conducts a research. The guideline starts from proposal preparation up to data collection and data processing focusing on ethical protocols. In the current study all methods starting from proposal preparation up to the final research was commented and edited by the institutional research reviewer boards of the university. Besides, all methods were corrected, edited and implemented in accordance with the relevant ethical protocols, guidelines and regulations of the university.

Informed consent: This research was conducted in Assosa Zone West of Ethiopia, on secondary school students. Assosa Zone Education office is the legal guardian of the school students. Therefore, formal informed consent was obtained from the Assosa Zone education office principals during the time of ethical approvals (Reference number: ASZ/EO/1039/12). Additionally, verbal consent was obtained from school directors and supervisors for distribution of questionnaires and data collection on students. After that, school directors, supervisors and students were collected into a meeting hall they had been told about the aims of the study, duration of the study, the type and number of participants needed, how confidentiality and privacy would be ensured. Moreover, besides, the participants also were assured that they have full right to participate or withdraw from the study. Confidentiality was kept at each step of data collection and processing. The participants did not receive any direct benefit or compensation for participating in the study.

\section{Acknowledgments}

The author acknowledges the Institutional Research Review committees of Assosa University for their research protocol approval and facilitating. I thank you Assosa University, College of education and behavioral Sciences for their financial funding during the study. I also acknowledged Assosa Zone education office for their permission to conduct the study in each school. I am also grateful to all school directors and supervisors for their help during data collection. I also acknowledged all participants who agreed to participate in this study. At last not least, I would like to grateful thanks the editor of the manuscript.

\section{Availability of data and materials}

Data available from the corresponding author on request

\section{Author contribution}

The author involved in the study design, data collection, analysis, and writing of the article. The author also read and approved the manuscript.

\section{Funding}


This study was funded financially by the College of Educational and behavioral Sciences at Assosa University in Ethiopia (Reference number: /RD/3095/12). The funding body has no input in the design of the study, data collection, analysis, and interpretation of data.

\section{Consent of publication}

Not applicable

\section{Competing interests}

The author declares that they have no competing interests.

\section{Author details}

Demelash Wubet, Woldia University, Ethiopia, College of Educational and Behavioral Sciences, Department of Early Child hood Care and Education

\section{References}

1. Clasen D \& Brown B. The multi - dimensionality of Peer Pressure in Adolescence. Journal of Youth and Adolescence. 1985; 14, 451- 468.

2. Morrison T, Kalin R \& Morrison M. Body-image Evaluation and Investment among Adolescents: A test of socio - cultural and social comparison theories in Adolescence. 2004; 39, 571 - 592.

3. Gonzales N. Family and Peer Influences on Adolescent Behavior and Risk-Taking. Arizona State University. 2010; 52- 61.

4. Alemnesh D. Negative Peer Pressure among Adolescent Students: (MA Thesis). Addis Ababa University, Ethiopia. 2014; 45-79.

5. Cole M \& Cole R. The Development of Children $\left(4^{\text {th }}\right)$. University of California, San Diago: worth publishers. 2001; 345-678.

6. Dishion T, Bullock B \& Gravic I. Pragmatism in modeling peer influence: Dynamics, outcomes, and change processes. Development and Psychopathology. 2002; 14, 969-981.

7. Rutgers W\& Labouvie E. Toward the assessment of adolescent problem of drinking alcohol (1989) http://eib.emcdda. europa.eu/index. cfm? fuseaction = Content \&None ID $=4200 \&$ Language ISO = E. Retrieved Feb.9, 2020.

8. Belay E. Factors contributing to sexual violence against female high school students. Addis Ababa University, Ethiopia. 2008; 53-68.

9. Amsale C \& Yemane B. Peer pressure is the prime driver of risky sexual behavior among school adolescents in Addis Ababa, Ethiopia. 2012; 34-89.

10. Meash G, Amsalu F. \& Tesfahun M. Assessment of Alcohol consumption Risky Sexual Behavior. Addis Ababa University, Ethiopia; 2010; 34-87. 
11. Tilaye N. Peer Group Influence, Alcohol Consumption and Academic Self Esteem of Adolescents: (MA Thesis). Addis Ababa University, North - Shoa Zone.2014; 51-57.

12. Nebiyou T. The Relationship between Peer Group Pressure and Responsibility Behavior (MA Thesis).Addis Ababa University, Ethiopia.2015; 59-65.

13. Burack R. Teenage sexual behavior: Attitudes towards and declared sexual activity. The British journal of family planning. 1999; 24 (4), 8-145.

14. Kandel D \& Andrews K. Processes of adolescent socialization by parents and peers. International Journal of Addictions.1987; 22, $319-342$.

15. Carter B \& McGoldrick M. The expanded life cycle: Individual, family, and social perspectives ( $3^{\text {rd }}$ ). Needham Heights, MA: Allyn \& Bacon.2005; 456-642.

16. Smith T. Adolescent gender differences in time alone and time devoted to conversation. Adolescence.1997; 83-87.

17. Kothari CR. Research Methodology: Methods' and Techniques $\left(2^{\text {nd }}\right)$.New Delhi: New Age International Ltd publishers;2004;342-456. 